\title{
Reply to 'Revisiting photocarrier lifetimes in photovoltaics'
}

Pivrikas et al. reply - We agree with

Stranks and Petrozza that long photocarrier lifetimes should be sought ${ }^{1}$. From the device performance perspective, the photocarrier lifetime needs to be longer than the extraction (transit) time to ensure lossless charge transport ${ }^{2}$.

Previously, we addressed the recombination differences between Langevin and non-Langevin systems ${ }^{3}$. In some systems, the majority charge carrier density is well known (for example, doping density in silicon) and is commonly reported together with the lifetime ${ }^{4}$. Only under these circumstances, when the density is measured, is a lifetime comparison appropriate. We agree that perovskites can be considered non-Langevin systems, but the relationship between density and lifetime applies regardless of the Langevin or non-Langevin nature of the system.
To conclude, it is erroneous to compare lifetimes if possible changes in carrier density are not accounted for. Illumination conditions close to solar intensity do not guarantee identical charge carrier density. A longer lifetime might simply indicate a reduced density, for example, because of a compromised quantum efficiency, decreased light absorption or photocarrier density redistribution in the material. In the interests of clarity, we wish to restate our initial remark that, in general, photocarrier lifetime alone should not be an optimization goal used to tune device fabrication parameters or to compare the recombination mechanisms in different systems.

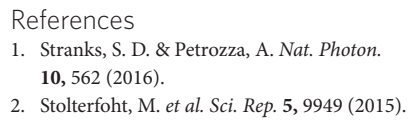

3. Pivrikas, A., Philippa, B., White, R. D. \& Juska, G. Nat. Photon. 10, 282-283 (2016).

4. Schroder, D. K. Semiconductor Material and Device Characterization (John Wiley \& Sons, 2006).

Almantas Pivrikas ${ }^{1,2 \star}$, Bronson Philippa ${ }^{3}$, Ronald D. White ${ }^{4}$ and Gytis Juška ${ }^{5}$ ${ }^{1}$ School of Chemistry and Molecular Biosciences and School of Mathematics and Physics, The University of Queensland, Brisbane 4072, Australia. ${ }^{2}$ School of Engineering and Information Technology, Murdoch University, Perth 6150, Australia. ${ }^{3}$ College of Science and Engineering, James Cook University, Cairns 4870, Australia. ${ }^{4}$ College of Science and Engineering, James Cook University, Townsville 4811, Australia. ${ }^{5}$ Department of Solid State Electronics, Vilnius University, 10222 Vilnius, Lithuania.

*e-mail: a.pivrikas@murdoch.edu.au 